\title{
Where will the concept of lymph node micrometastasis advance?
}

\author{
Shoji Natsugoe
}

Received: 15 May 2013/Published online: 18 June 2013

(C) Japan Society of Clinical Oncology 2013

There are several morphological pathways by which lymph node metastasis can arise: invasion into a deeper layer; detachment of tumor cells from the primary tumor; infiltration into intramural lymphatics; flow to extramural lymphatics; arrival to afferent lymphatics of a marginal sinus; movement to the lymph node cortex; and implantation of tumor cells into the node and formation of metastasis. Some cancer cells move from an efferent lymphatic vessel to the next lymph node. In terms of the first step toward lymph node metastasis, a small number of cancer cells is thought to be related to the formation of metastasis. Recent research into biological aspects has elucidated various tumor characteristics. What kinds of tumor cells have metastatic potential? Many molecules in the pathways toward lymphatic metastasis are thought to be related. Once tumor cells invade the lymphatics, can all cells implant into lymph nodes? Numerous immune cells are originally present in the lymph nodes, and such cells fight and defend against the invasion of bacteria, viruses, tumor cells, and so on. Thus, it is speculated that only certain special cancer cells can implant and grow to form an overt metastasis. What are these special cancer cells? The recent concept of epithelial-mesenchymal transition and the stem cell theory seems to offer an important key to solving the properties of tumor cells. Tumor cells able to seed a metastasis must presumably have the ability to avoid immune defense cells. Further basic studies will elucidate the mechanisms underlying lymphatic metastasis.

S. Natsugoe $(\bowtie)$

Department of Digestive Surgery, Breast and Thyroid Surgery,

Kagoshima University Graduate School of Medical and Dental

Sciences, 8-35-1 Sakuragaoka, Kagoshima 890-8520, Japan

e-mail: natsugoe@m2.kufm.kagoshima-u.ac.jp
Meanwhile, in the clinical field, lymph node metastasis offers an important prognostic factor for gastrointestinal (GI) cancer. The presence of tiny lymph node metastasis, in the form of lymph node micrometastasis (LNM) that is not detectable by routine histological examination, has been reported in carcinomas of various organs. Although overt lymph node metastasis is thought to be related to prognosis, what is the relationship between LNM and prognosis? Recent advances in techniques such as immunohistochemistry (IHC) and reverse transcription-polymerase chain reaction (RT-PCR) have allowed the detection of LNM. Recently, LNM has been defined by the criteria of TNM classification and roughly divided in some categories according to the size of the metastatic foci or the method of detection. Clinical evaluation of LNM is somewhat difficult, because of the differences in study designs and methods of detection, such as the sample size, tumor stage of patients, number of removed nodes based on the area of lymph node dissection, use of immunohistochemistry, RTPCR, or other methods, and the kinds of antibodies or primers used. Several articles have discussed the clinical significance of LNM of GI cancer.

The present review articles summarize the current knowledge of LNMs in GI cancer from the perspectives of both molecular and biological characteristics and clinical aspects. These articles will be helpful as an overview of the current understanding of LNM and areas requiring further investigation.

Conflict of interest The author declares that he has no conflict of interest. 